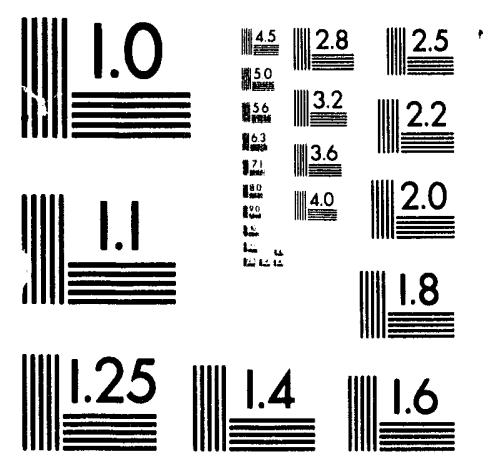



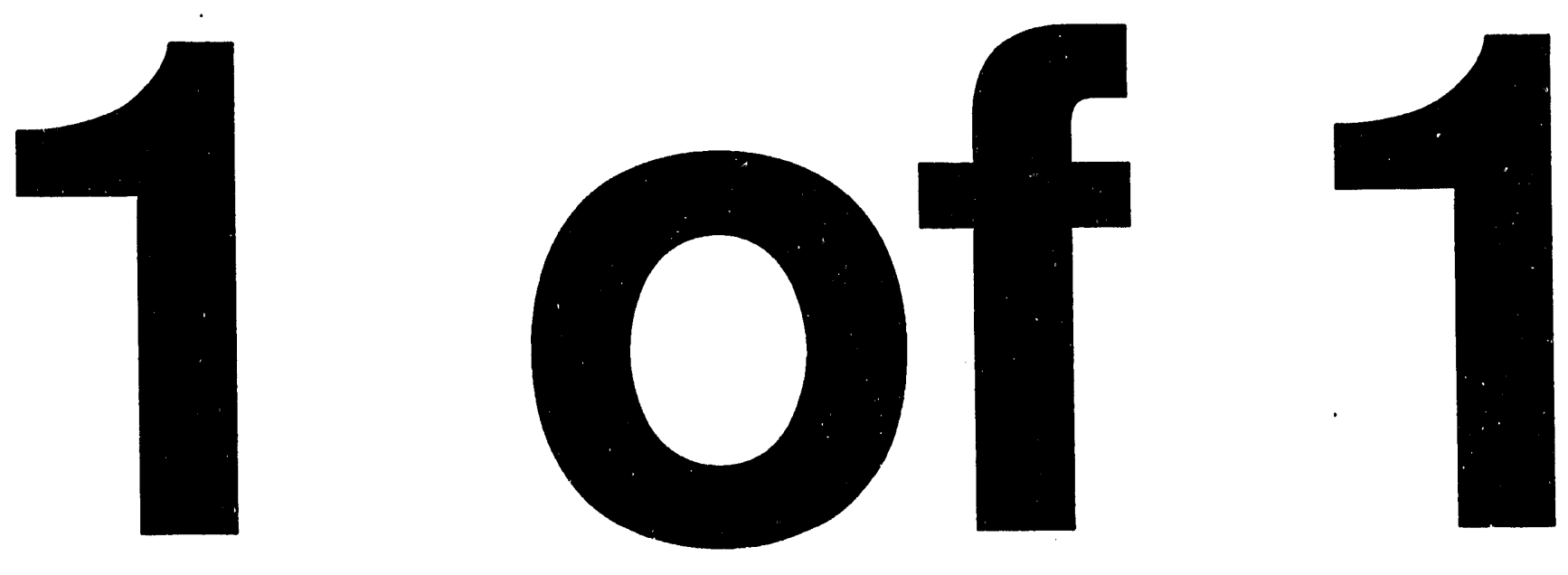


\title{
WATER-COOLED ION-MILLED DIFFRACTION GRATINGS FOR THE SYNCHROTRON RADIATION COMMUNITY*
}

\author{
Wayne R. McKinney \\ Advanced Light Source \\ Accelerator and Fusion Research Division \\ Lawrence Berkeley Laboratory \\ University of California \\ Berkeley, CA 94720 \\ and \\ Clayton L. Shannon and Earl N. Shults \\ Electro-Optical Systems Division \\ Hughes Aircraft Company \\ El Segundo, CA 90245
}

August 1993

Paper presented at the 8th National Conference on Synchrotron Iadiation Instrumentation Gaithersburg, MD, August 23-26, 1993

\section{MASTER}

DISTRIBUTION OF THIS DOCUMENT IS UNLIMITED

*This work was supported by the Director, Office of Energy Research, Office of Basic Energy Sciences, Materials Sciences Division, of the U.S. Department of Energy, under Contract No. DE-AC03-76SF00098. 


\title{
Water-cooled Ion-Milled Diffraction Gratings for the
}

\section{Synchrotron Radiation Community*}

\author{
Wayne R. McKinney \\ Advanced Light Source, Accelerator and Fusion Research Division \\ Lawrence Berkeley Laboratory \\ Berkeley, CA 94720
}

Clayton L. Shannon and Earl N. Shults

Electro-Optical Systems Division

Hughes Aircraft Co.

El Segundo, CA 90245

\section{Introduction}

Key technical and strategic choices are reviewed, leading to the fabrication method of ion-milled grating grooves for the monochromators at the Advanced Light Source (ALS) at Lawrence Berkeley Laboratory (LBL), and for other synchrotrons. Several laboratories and their industrial partners have joined to manufacture gratings with essentially theoretical performance. Metrology data and theoretical comparisons are given for square wave profile grating samples ion-milled into electroless nickel surfaces. The extensive capabilities of Hughes Aircraft in grating manufacture are reviewed.

* This work was supported by the Director, Office of Energy Research, Office of Basic Energy Sciences, Materials Sciences Division of the U.S. Department of Energy, under Contract No. DE-AC03-76SF00098. 


\section{History}

The history of manufacture of holographically patterned grating grooves that are ion-milled into the substrate material is one of many problems for the synchrotron radiation community. Often the delivery times have been unreasonably long; four years is a frequently heard story. We personally know of one researcher who has never had an ion-milled grating delivered while he was still working at the lab that placed the order. Quality has been extremely variable. Some gratings were very uniform and some had only a small percentage of usable groove area. Exotic materials were not available, which was reasonable, since often the vendor was still smarting from his losses on gratings made on more conventional materials.

All of these problems were compounded by the lack of suitable metrology to measure the grating blanks. Justifiably, the vendors were reluctant to fabricate grooves onto blanks made by some other vendor, since there was little agreement on optical specifications for the grating blank or on methods of measurement. Finally, most of the available vendors had little depth; they were of ten limited by having only one skilled engineer or only one laser.

Ruled gratings were often substituted for ion-milled gratings. These kinds of gratings share many of the same problems listed above. In addition, the ruling diamond can wear out over the course of the ruling, limiting the gain in efficiency from the blaze effect. Quality is often variable. Even if ruled extremely well, ruled gratings have in 
principle more higher order contamination when used in monochromators than do square wave profile gratings.

The ALS chose a fully integrated vertical approach to the problems of grating and grating blank procurement. Early in the project (1986) it was decided to limit the optical components to the combination of GlidcopTM for the cooled body of the optic and electroless nickel for the polishable layer. ${ }^{1}$ Silicon carbide was briefly considered, but was rejected as too early in development for the inclusion of cooling channels without costly developmental efforts. Together with Mr. David Lunt of Tucson Optical Research Corp. (TORC) and later of Photon Sciences (both of Tucson, AZ), the ALS developed an excellent source for the electroless nickel plating: Acteron Metal Finishers of Redwood City, CA. The electroless nickel coating which had previously been polishable to only about 10 Angstroms RMS can now often be polished to better that two Angstroms RMS. Sometimes this is not achieved, due to irregularities in the plating, but three to four Angstroms is routine. $2-3$

Simultaneously with this development, the ALS invested considerable resources in the manufacture of two Takacs style long trace profiler instruments (LTP II) in cooperation with Brookhaven National Lab (BNL), Continental Optical, and Baker Engineering.4-5 One of the LTP II's remains at LBL and the other, originally at TORC/PHOTON, is now at Rockwell Power Systems (RPS) in Albuquerque, NM. RPS has licensed the polishing of the nickel and has combined it with their considerable experience in the 
manufacture of silicon optics for the laser weapons effort. 6 RMS slope errors of 1.0 micro-radian over 12 inches have been obtained on these water cooled metal mirrors. These results have been confirmed on long radius spherical parts by novel interferometric metrology at RPS. In addition RPS has invested in the development of a large (145") continuous polishing machine.

\section{Grating Grooves}

With this excellent foundation of well-characterized grating blanks the ALS approached the problem of grating groove fabrication from the standpoint of finding a source that would not jeopardize the $\$ 100 \mathrm{M}$ already invested in the ALS. Hughes Aircraft Company has a long history in the manufacture of square wave grating grooves for the laser weapons program. They routinely manufacture gratings with a period of one quarter micron (2500 Angstroms) to thirty (30) microns. They have a photoresist processing and ion-milling capability for the fabrication of photolithographically (holographically) patterned gratings up to two meters in size. Figure 1 shows typical uses of their capability. The high energy laser beam, generated in a turbulent chemical laser cavity, requires active feedback to stabilize the wavefront. Very shallow square wave grooves are used to diffract a small portion of the energy to a wavefront sensor that feeds back to a deformable mirror. More efficient gratings are required earlier in the optical path as aperture sharing elements that reflect the high energy laser, and diffract a different wavelength laser beam to a tracking system. Considerable federal investment has created this extensive capability at Hughes 
over the last two decades. ${ }^{7-8-9}$ Hughes currently has a $\$ 12 \mathrm{M}$ contract for the construction of the ALI/HOE (ALPHA LAMP Integration/Holographic Optical Elements) gratings that consist of 200 focusing holographic gratings spread over a segmented mirror device several meters across. Hughes has considerable depth in equipment and trained personnel that is not available at other grating manufacturers.

\section{Samole Gratings}

In order to assure that the gratings fabricated at Hughes were suitable for use in the soft X-ray region, LBL ordered two sample gratings from Hughes. These were fabricated on two 2" diameter witness coupons which had been plated with electroless nickel, and polished simultaneously with an ALS water-cooled optical component. These gratings were received within the specified eight week delivery time, and were nominally specified to have 1000 grooves per mm, 100 to 120 Angstroms groove depth, and a 50-50 groove top to bottom ratio. These gratings were measured at the Center for X-ray Optics (CXRO) at LBL,10 and at Daresbury Laboratory in the United Kingdom. ${ }^{11}$ Figure 2 shows a detector scan made at the CXRO of one of the sample gratings made at 110 Angstroms wavelength using a laser plasma soft X-ray source. Clearly demonstrated are extremely low stray light, phase cancellation in the zero and second orders, and good symmetry between the inner and outer first orders. Figure 3 shows data taken at Daresbury Laboratory at $278 \mathrm{eV}$ photon energy. These data compare very well with the plotted theoretical efficiency using the Nevière theory. 
Excellent performance was also obtained at $400 \mathrm{eV}$ photon energy. Figure 4 shows an SEM photograph of these grating grooves, and Figure 5 shows an SEM photograph of the photoresist lines used to mask the nickel surface for ion-milling. The mask is seen to be extremely clean and uniform. This uniformity was confirmed in a scanning measurement of the diffraction efficiency at CXRO. In one configuration the grating was observed to have an absolute efficiency of $15 \% \pm 1 \%$ over the whole area of the grating.

\section{The Collaboration}

In order to make a significant size order for Hughes (on the order of one half million dollars), several laboratories and companies joined together on the grating project. Efim Gluskin of the Advanced Photon Source (APS) at the Argonne National Laboratory (ANL) contributed $\$ 30 \mathrm{~K}$ for the purchase of a $6^{\prime} \times 16^{\prime}$ vibration isolated optical table. LBL contributed $\$ 22.4 \mathrm{~K}$ for the construction of an acoustically damping cover for the table. Photon Sciences agreed to manufacture two large off-axis paraboloids and two large folding flats for the holographic grating setup. In addition, Photon Sciences would apply an anti-reflection coating to the blanks to prevent standing waves in the photoresist from affecting the photolithography. In this way the community could have a dedicated setup, and never have to wait in line for a table behind a much larger military order.

On the basis of the excellent sample gratings described in the previous section, the following grating orders were solicited. ESRF is 
the European Synchrotron Facility. "U" indicates an undulator source, and "BM" indicates a bending magnet source.

\begin{tabular}{|c|c|c|c|c|}
\hline Laboratory & Beamline & Number & Substrate & Polisher \\
\hline LBL & U5, BL 7.0 & 3 & ENi/Glidcop & Photon \\
\hline LBL & U8, BL 9.0 & 3 & ENi/Glidcop & Photon \\
\hline LBL & BM BL 9.3.2 & 1 & ENi/Glidcop & Photon \\
\hline IBM & U5 BL 9.0.1 & 3 & ENi/Glidcop & Rockwell \\
\hline ESRF & & 3 & $\mathrm{SiC}$ & Rockwell \\
\hline APS & future SGM & 2 & Silicon & Rockwell \\
\hline
\end{tabular}

The blanks for IBM are finished, and the blanks for ESRF are in final polishing. There was considerable difficulty in the procurement of the solid chemically vapor deposited (CVD) silicon carbide $(20 \mathrm{~mm}$ thick). The vendor had to repeatedly re-fabricate the blanks to obtain good material without defects. Even now the polishing is taking much longer than predicted, and we are not enthusiastic about the solid CVD SiC material. To reduce costs in the calibration of the ion-milling and anti-reflection coatings, sputtered nickel will serve as the ion-milled layer on the silicon carbide and silicon grating blanks.

\section{Current Progress}

Figure 6 shows the holographic table with the acoustically damping cover partially completed. It is now finished. Unfortunately, Photon Sciences has had some difficulty in the manufacture of some of the 
very high quality optics for the holographic setup. Hughes has been able to substitute smaller off-axis paraboloidal mirrors and folding mirrors that will allow us to start some of the seven planned groove frequencies from $150 / \mathrm{mm}$ to $2100 / \mathrm{mm}$. We anticipate that the first exposures and ion milling will occur in the next few weeks. Figure 7 shows a duty cycle calibration made on the new table and setup, and figure 8 displays Twyman-Green interferograms showing the diffracted wavefront quality of a test grating fabricated from the initial optical alignment. The wavefront quality is very good, and should improve significantly with final survey and alignment of the table. The support staff at Hughes can perform calculations from the interferogram, and specify the necessary adjustments to the optical setup.

\section{Conclusion}

We have reviewed the collaborative project for ion-milled gratings for the synchrotron community. Even though we have experienced some minor setbacks, we fully expect to fabricate diffraction grating grooves with theoretical performance in time to not significantly impact the scheduling for the ALS monochromators and the projects of our other customers. After these gratings are complete and tested, we plan to fabricate future gratings in smaller lots, and at less cost once the optical setup is finished, and all of the costs are well characterized. 


\section{Acknowledgments}

Any project of this magnitude necessarily consists of a number of people whose contributions are essential to its success. At Photon Sciences the polishing is done by David Lunt, President, Eric Palmer, General Manager, and Ron Appels, Master Optician. The antireflection coatings are the work of Greg Lowe, Coating Engineer. At RPS we wish to mention Bill Lowrey, Program Manager, Tom Tonnessen, Mechanical Engineer, and John Bender and Don Ewing, Master Opticians. The original design of water-cooled GlidcopT M substrates was by Dick Digennaro and Tom Swain, Staff Scientists in Mechanical Engineering at LBL. Blank metrology at LBL and LTPII development were the effort of Steve Irick, Staff Scientist in Mechanical Engineering at LBL. The measurements of the efficiency at LBL were by Eric Gullikson, Staff Scientist in the CXRO and Phil Heimann, Staff Scientist in the ALS. Efficiency measurements at Daresbury Laboratory were by Howard Padmore, now ALS Experimental Systems Group Leader. The ion-milling of both the samples and the final gratings are the product of Hugh Garvin and Klaus Robinson of Hughes Malibu Research Labs. Finally we must thank our financial contributors and customers, LBL, APS (Efim Gluskin, Group Leader, Insertion Devices), IBM (Jo Stöhr, Franz Himpsel, Mahesh Samant), and ESRF (Nick Brookes). 


\section{Mckinney et al figure captions}

Figure 1: This optical diagram shows typical applications of Hughes' ion-milled square wave profile diffraction grating capability.

Figure 2: Detector scan of diffracted energy from Hughes grating sample using the $\mathrm{X}$-ray plasma source at the Center for $\mathrm{X}$-ray optics at LBL.

Figure 3: This graph shows the diffraction efficiency for the $-1,0$, and +1 orders as a function of the grazing angle of incidence for a photon energy of $278 \mathrm{eV}$ together with the best fit obtained with the Nevière model.

Figure 4: This micrograph shows the actual sample grating grooves etched into the nickel substrate.

Figure 5: These micrographs show a test exposure series used to calibrate the line width ratio.

Figure 6: This shows the large vibration isolated table, and the initial construction of the acoustically damping cover.

Figure 7: This micrograph shows a test run of photoresist masking with the latest optical setup.

Figure 8: These are Twyman-Green interferograms showing diffracted wavefront quality of test grating exposures made with the initial setup of the large table. 
1 DiGennaro, R., Gee, B., Guigli, J., Hogrefe, H., and Howells, M.R., "A Water Cooled Mirror System for Synchrotron Radiation," Nuclear Instruments and Methods in Physics Research A266 (1988) 498.

2 McKinney, Wayne R., Irick, S. C., and Lunt, D. L. J., "Water Cooled Metal Optics for the Advanced Light Source," 7th National Conference on Synchrotron Radiation Instrumentation, Baton Rouge, LA, October 28-31, 1991, Nuclear Instruments and Methods in Physics Research A319, 1992, pp 179-184. (LBL\#31259)

3 McKinney, Wayne R., Irick, Steven C., and Lunt, David L. J., "XUV Synchrotron Optical Components for the Advanced Light Source: Summary of the Requirements and the Developmental Program," SPIE Proceedings, Vol. 1740, Optics for High Brightness Synchrotron Beamlines, 1992, pp. 154-160.

4 Irick, S. C., McKinney, W. R., Lunt, D. L. J., and Takacs, P. Z., "Using a Straightness Reference in Obtaining More Accurate Surface Profiles from a Long Trace Profiler," SRI-91, The 4th International Conference on Synchrotron Radiation Instrumentation, Chester, U.K. 15-19 July '91, Review of Scientific Instruments. Vol. 63 No 1 (Part IIB), Jan. 1992. pp 1436-1438.

5 Irick, S. C., "Determining surface profile from sequential interference patterns from a long trace profiler" SRI-91, The 4th International Conference on Synchrotron Radiation Instrumentation, Chester, U.K. 15-19 July '91, Review of Scientific Instruments, Vol. 63 No 1 (Part IIB). Jan. 1992, pp 1432-1435.

6 Lunt, D. L., Bender, J. W., Ewing, D. G., and McKinney, W. R., "XUV Synchrotron Optical Components for the Advanced Light Source-Fabrication and Metrology," SPIE Proceedings, Vol. 1740, Optics for High Brightness Synchrotron Beamlines, 1992, pp. 161-172.

$7 \mathrm{Au}$, Anson and Garvin, Hugh L., "Holographic surface grating fabrication techniques," SPIE Proceedings, Vol. 240, Periodic Structures, Gratings, Moiré Patterns, and Diffraction Phenomena, 1980, pp. 13-17. 
8 Garvin Hugh L., Au, Anson, and Minden, Monica L., "Ion-etched grating for laser applications," SPIE Proceedings, Vol. 240, Periodic Structures, Gratings, Moiré Patterns, and Diffraction Phenomena, 1980, pp. 63-68.

9 Worthington, R. J., Wu, N., and Spencer, H. M., "Design of holographic gratings on primary mirrors for beam sampling," SPIE Proceedings, Vol. 240, Periodic Structures, Gratings, Moiré Patterns, and Diffraction Phenomena, 1980, pp. 56-62.

10 Heimann. P. A., Gullikson, E. M., and McKinney, Wayne, R., "The Efficiency of a Blazed and a Laminar Grating," X International Conference on Vacuum Ultraviolet Radiation Physics, Paris, France, 27-31 July, 1992.

11 Howard Padmore, private communication. 


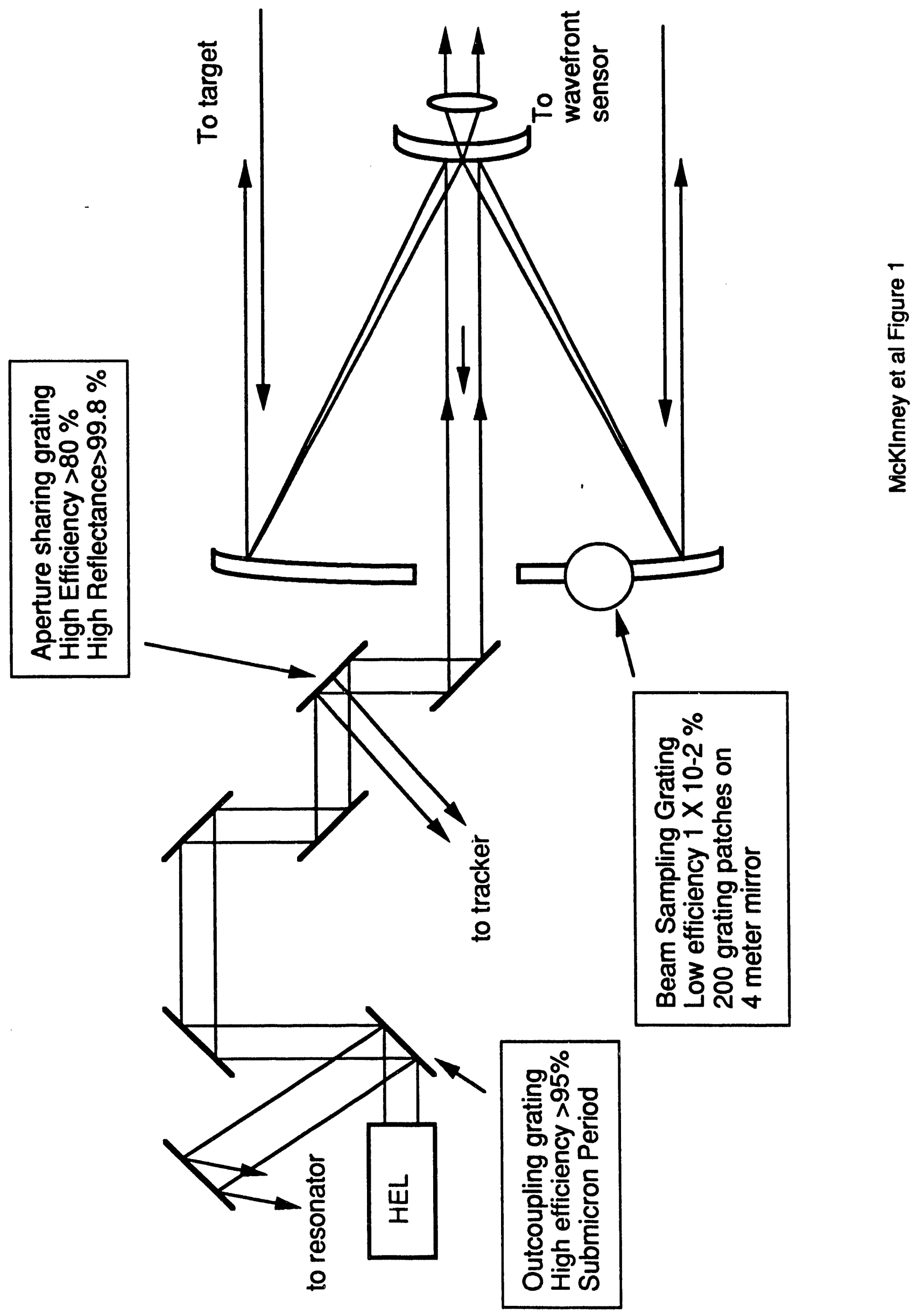




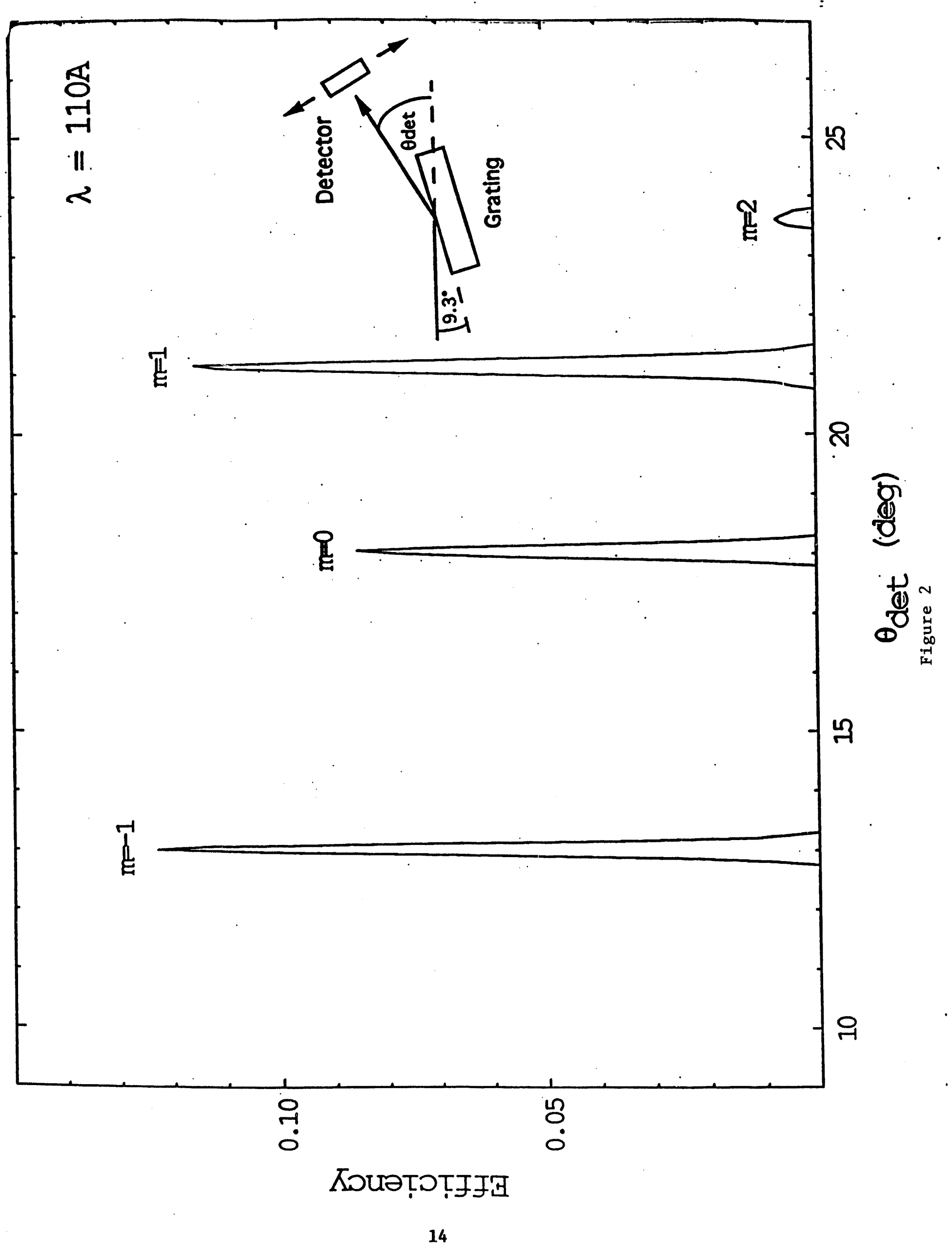




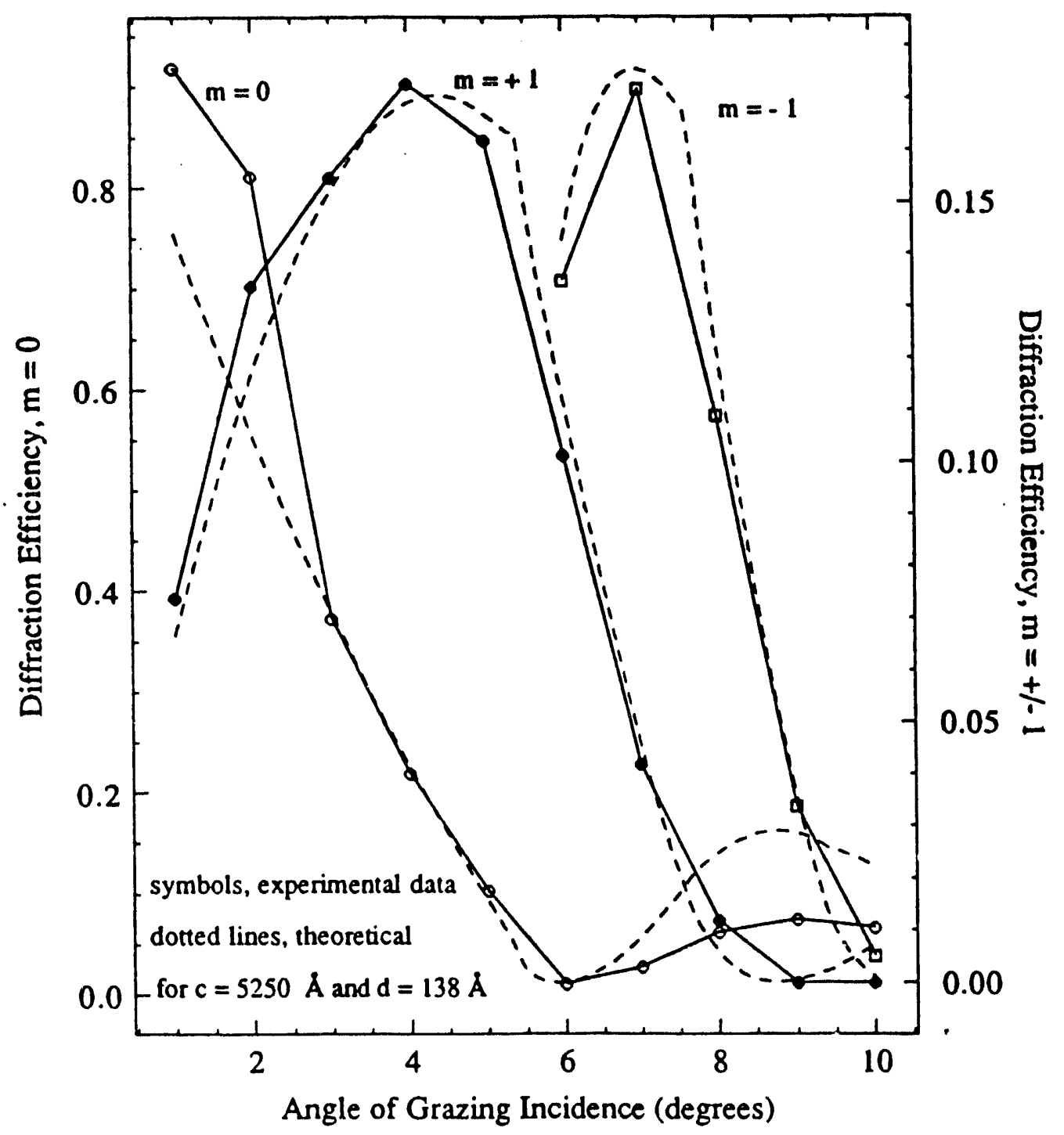

Figure 3 


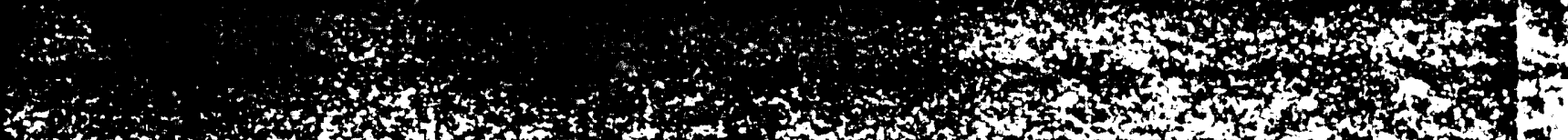

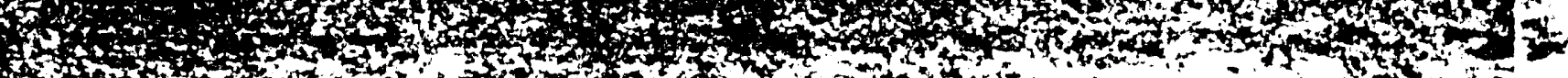

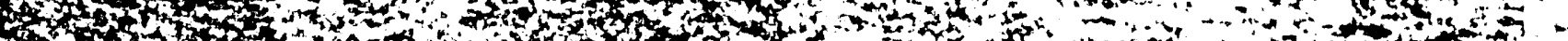

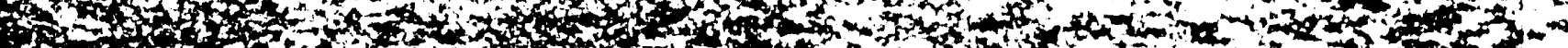

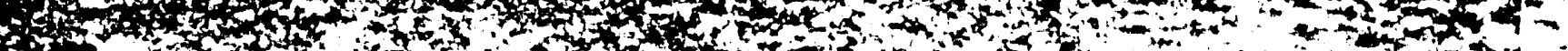

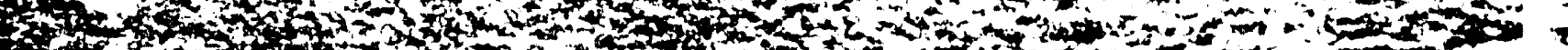

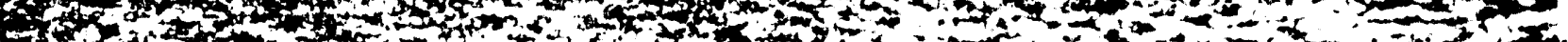

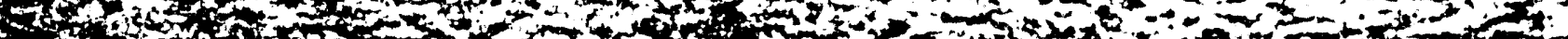

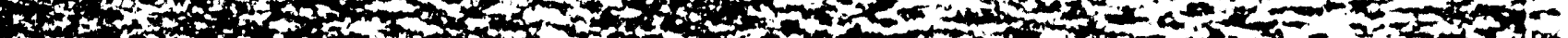

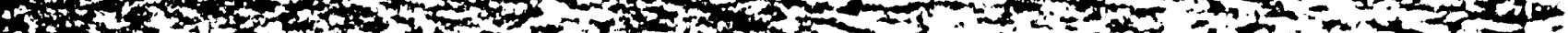

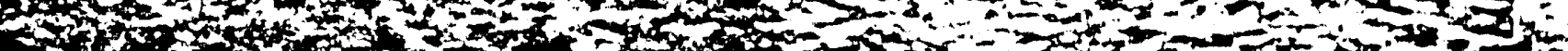

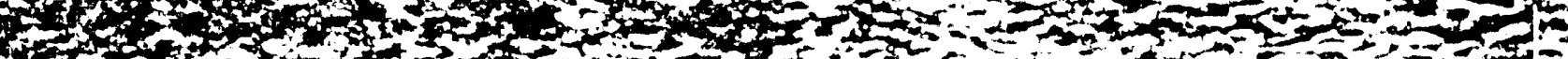

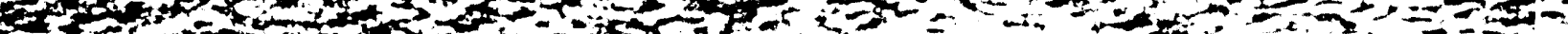

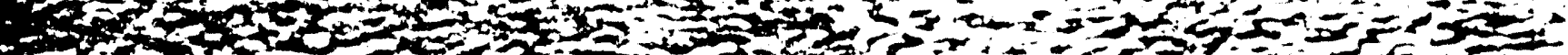

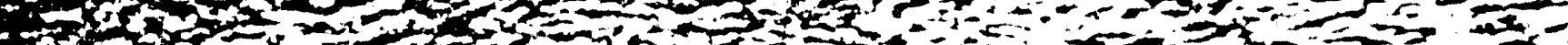

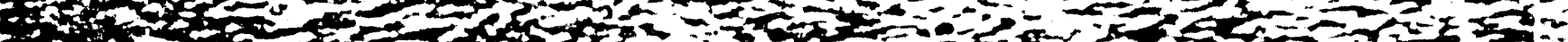

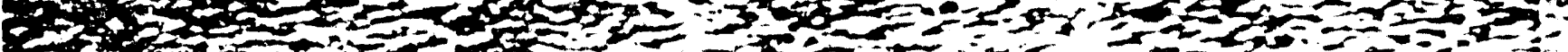

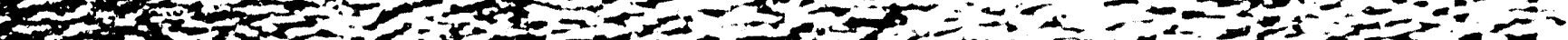

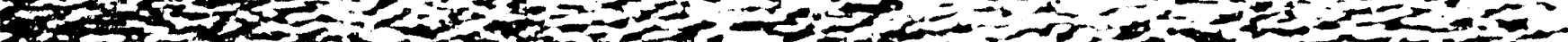

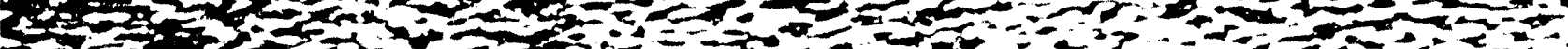

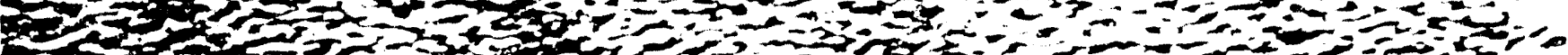

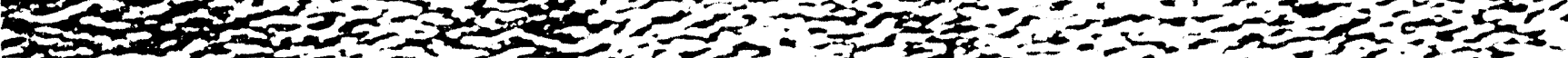

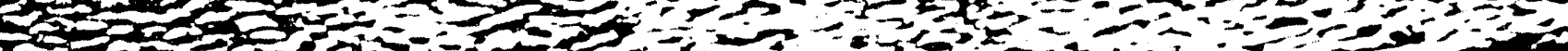

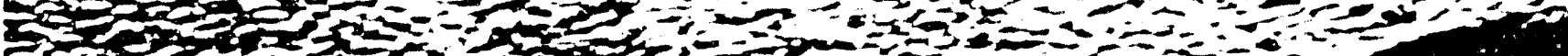

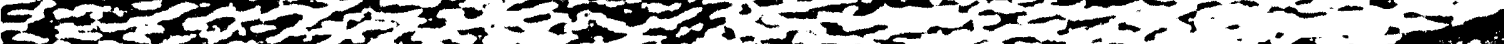

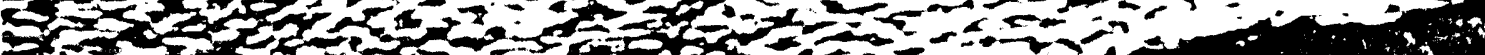

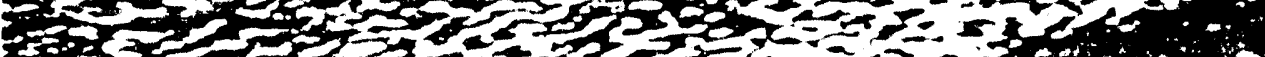

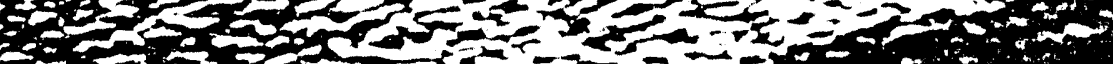

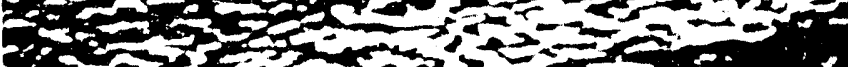

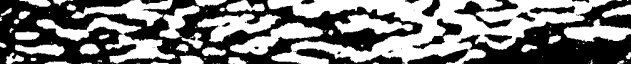
$x=25$

(2)

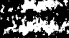

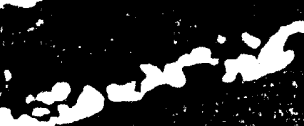

(n)

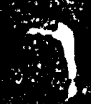

$\lim _{1}$
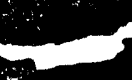

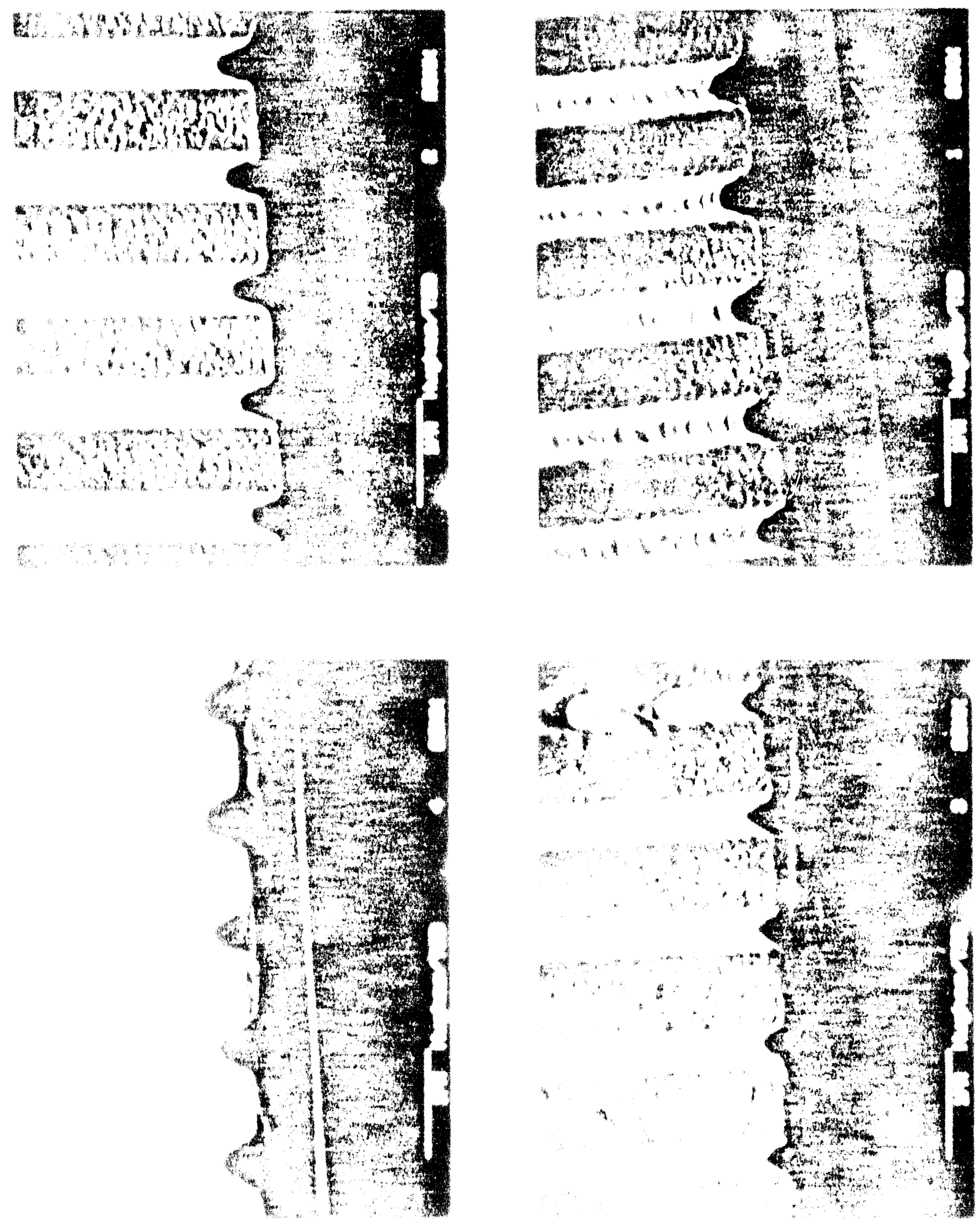


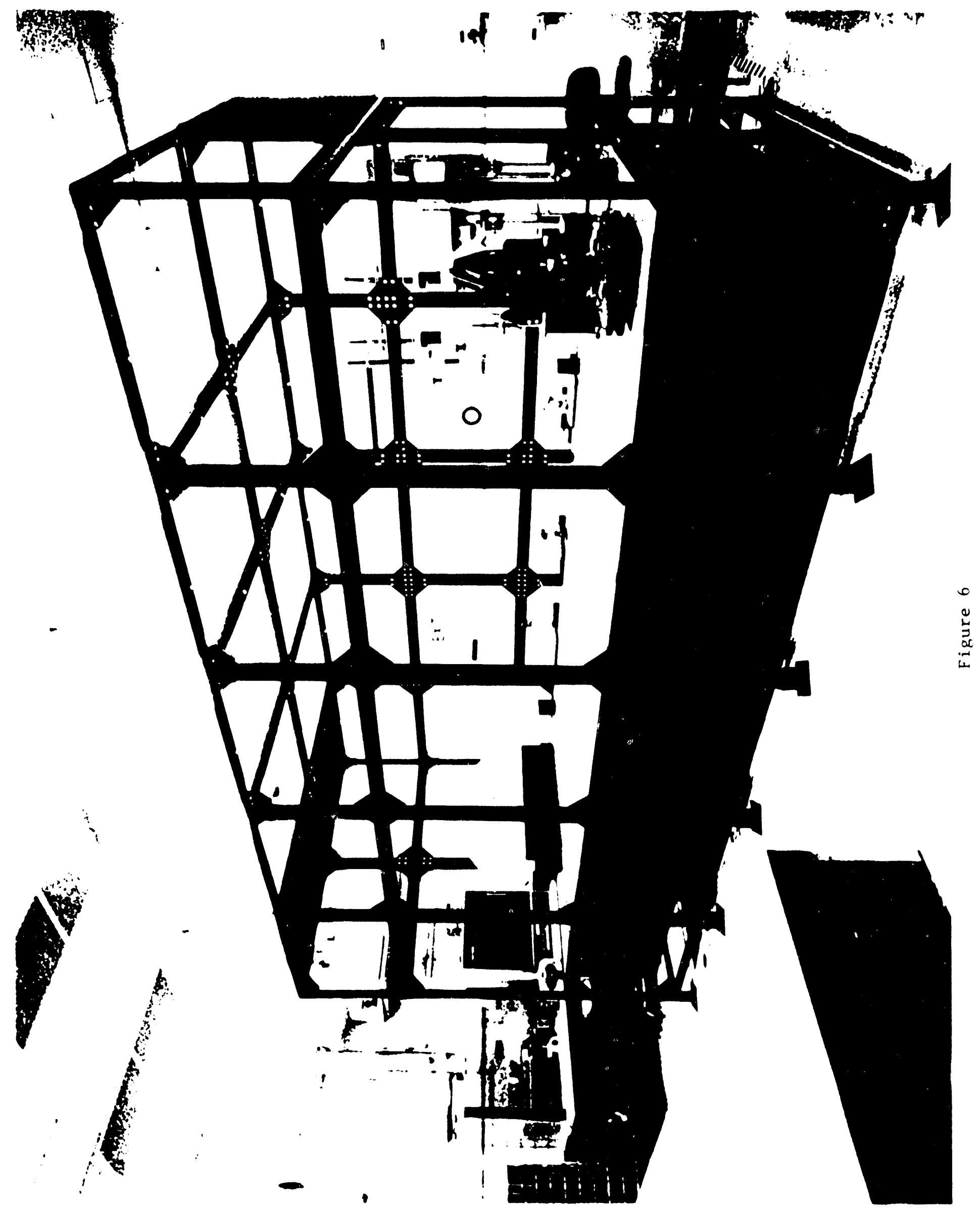




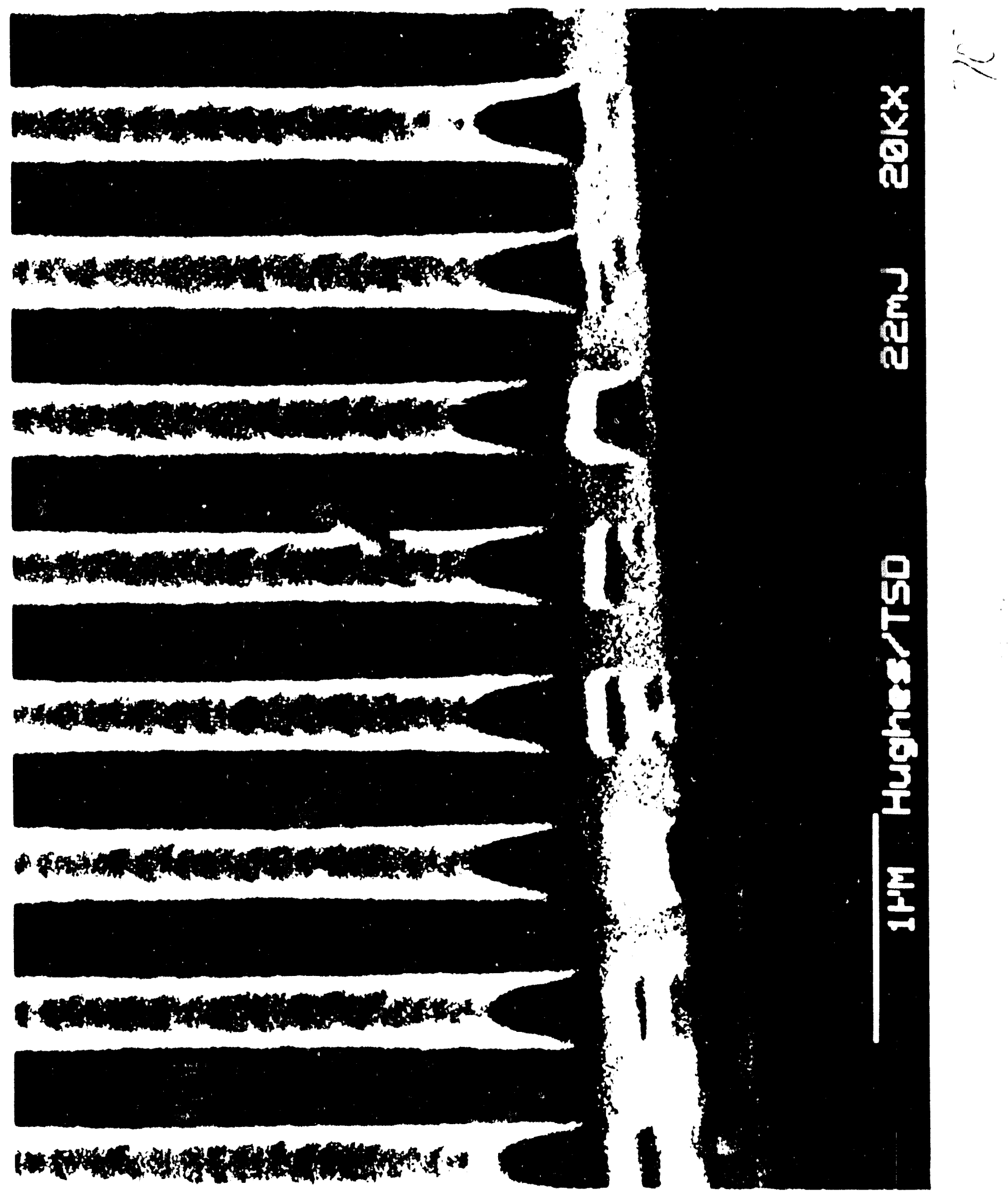




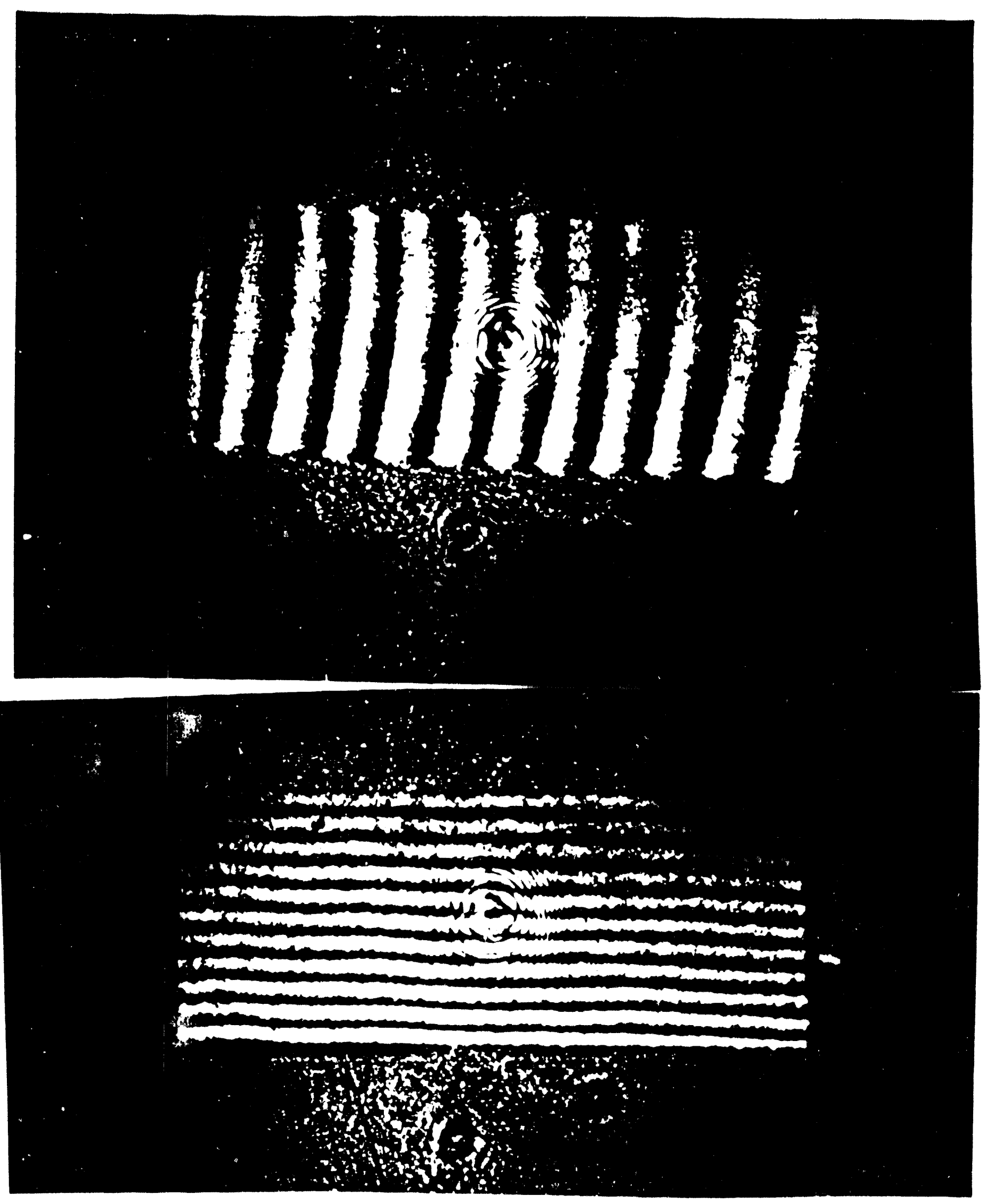



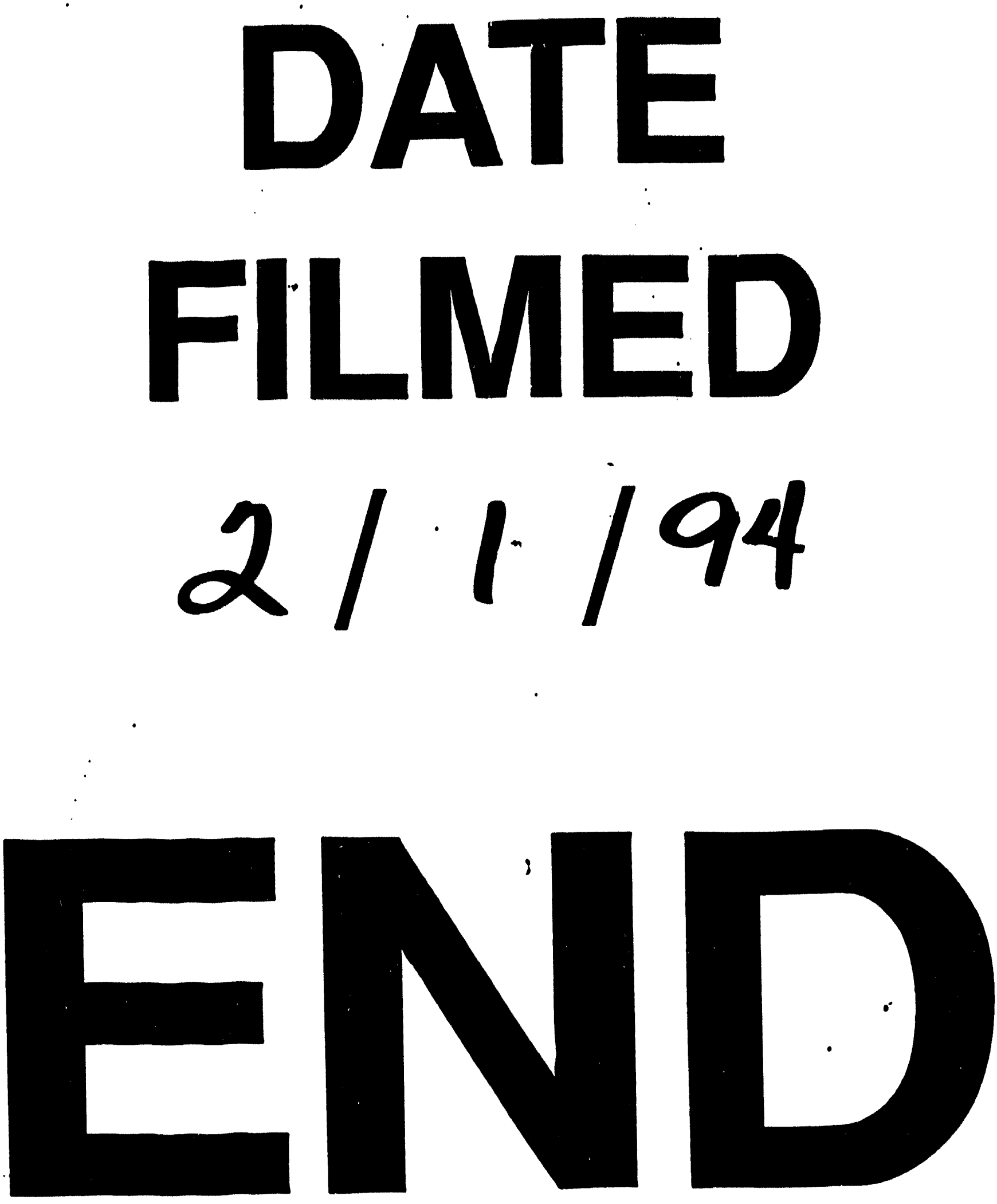


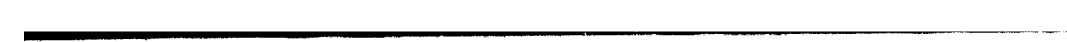

